\title{
Self-field effects in Josephson junction arrays
}

\author{
Petraglia, Antonio; Filatrella, G.; Rotoli, G.
}

\section{Published in:}

Physical Review B

Link to article, DOI:

10.1103/PhysRevB.53.2732

Publication date:

1996

\section{Document Version}

Publisher's PDF, also known as Version of record

Link back to DTU Orbit

Citation (APA):

Petraglia, A., Filatrella, G., \& Rotoli, G. (1996). Self-field effects in Josephson junction arrays. Physical Review B, 53(5), 2732-2738. https://doi.org/10.1103/PhysRevB.53.2732

\section{General rights}

Copyright and moral rights for the publications made accessible in the public portal are retained by the authors and/or other copyright owners and it is a condition of accessing publications that users recognise and abide by the legal requirements associated with these rights.

- Users may download and print one copy of any publication from the public portal for the purpose of private study or research.

- You may not further distribute the material or use it for any profit-making activity or commercial gain

- You may freely distribute the URL identifying the publication in the public portal

If you believe that this document breaches copyright please contact us providing details, and we will remove access to the work immediately and investigate your claim. 


\title{
Self-field effects in Josephson junction arrays
}

\author{
A. Petraglia \\ Physics Department, DTU, Dk-2800 Lyngby, Denmark \\ and Unità INFM and Dipartimento di Fisica, University of Salerno, I-84081 Baronissi (SA), Italy \\ G. Filatrella \\ Unità INFM and Dipartimento di Fisica, University of Salerno, I-84081 Baronissi (SA), Italy \\ and Physikalisches Institut, Lehrstuhl Experimentalphysik II, University of Tübingen, D-72076 Tübingen, Germany \\ G. Rotoli \\ Unità INFM and Dipartimento di Energetica, University of L'Aquila, I-67010 Località Monteluco-Roio Poggio (AQ), Italy
}

(Received 27 July 1995)

\begin{abstract}
The purpose of this work is to compare the dynamics of arrays of Josephson junctions in the presence of a magnetic field in two different frameworks: the so-called $X Y$ frustrated model with no self-inductance and an approach that takes into account the self-field generated by the screening currents (considering self-inductances only). We show that, while for a range of parameters the simpler model is sufficiently accurate, in a region of the parameter space solutions arise that are not contained in the $X Y$ model equations.
\end{abstract}

\section{INTRODUCTION}

Arrays of Josephson junctions have been proposed more than two decades ago to enhance the emission of microwaves. ${ }^{1}$ In fact, it is well known that the power available from a single junction is not enough for many practical applications, and therefore the achievement of coherent motion of arrays of junctions is an important issue for device applications. $^{2}$ Apart from the applications, two-dimensional arrays have been also investigated as an interesting nonlinear system both experimentally and theoretically. ${ }^{3,4}$ In the study of such arrays two classes of models have been proposed to describe the dynamics:

(1) Models that neglect the self-field effects. These model are often called "uniformly frustrated $X Y, " 5-7$ because the Hamiltonian for this model is similar to that of a square flat lattice of spins in the magnetic field (for that reason it is also called the spin-glass model).

(2) Models that include, to some extent, the self-field effects. The simplest version of this model assumes that the self-field is generated by a "screening current," which shields the magnetic field only in the two cells adjacent to each branch where a current is flowing (in the following this model will be termed the NS model after Nakajima and Sawada, who introduced $\mathrm{it}^{8}$ ). More generally, one should consider the effect of the screening in all cells (full mutual inductance approach ${ }^{9,10}$ ).

To neglect screening currents greatly simplifies the problem. Moreover, the formal similarity with other well-known systems allows us to take advantage of an accumulated experience in those contexts. Model (2) has been used less extensively also because, in its complete form (which includes all mutual inductances), it requires complex numerical routines, and the resulting equations are difficult to handle analytically. ${ }^{9,10}$ Nevertheless the comparison among the different forms has already been addressed. For instance Phillips et al. ${ }^{9}$ have investigated the static properties of the vortices; they have showed that the shape of a static fluxon in the NS is different from the shape of the static model in the full approach. Model (2) has also been used extensively to predict the magnetic behavior of two-dimensional arrays. ${ }^{10,11}$ Dominguez and Josè have reviewed the topic in Ref. 4. In spite of the simplicity of model (1), it has been able to explain most of the experimental observations on twodimensional arrays, for example, giant Shapiro steps. ${ }^{12}$ It is therefore of interest to establish the limits of validity of this approach that should be taken into account when interpreting the experimental data. Moreover, to develop coherent sources based on large two-dimensional arrays of Josephson junctions, better performance can be obtained if the junctions in the array have larger critical currents; in fact, the emitted power of a single junction is proportional to the square of the critical current, while the emitted power of a mismatched coherent array will be $\propto N^{2} I_{0}^{2}$, where $N$ is the number of locked junctions in the array. ${ }^{13}$ In the following it will be shown that screening currents are small when the coupling, proportional to the inverse of the critical current, is too strong. Therefore, model (2) seems more suitable to describe arrays usable as microwave sources.

The purpose of this work is to explore the parameter space looking for the limits of validity of model (1) rather than to focus on a specific experiment. We will show that, for certain ranges of the parameters and at least for the simplest arrays, the solutions of model (1) are different from the solutions of model (2). For the sake of simplicity, we will compare the results obtained only with the simplest version of model (2), the NS. However, it should always be borne in mind that in some cases solutions arising within the context of model (2) can be quite different. ${ }^{9}$ To fully understand the differences between the two approaches we will present a step-by-step derivation of the dynamical equations. As a tutorial example we will start with a discussion of a simple dc superconducting quantum interferometer device (SQUID) containing only two junctions. This well-known case will 
lead us to some general considerations on the role of the screening currents.

It will be shown that the $X Y$ equations cannot be easily derived from NS equations taking some limits of the parameters. It will be further shown numerically that there is a region of the parameter space where it is not possible to assume that the screening current is negligible. Next, we will consider the most elementary cell of a two-dimensional array, i.e., a square cell with junctions (and inductances) on each branch. ${ }^{14}$ We will again derive the equations, and we will numerically show that the screening currents are not negligible for some ranges of the parameters. A comparison with the actual two-dimensional arrays will be performed for some cases to check that the results for the single cell are reliable. Finally, the microscopic dynamics in the two regimes (with negligible and non-negligible screening currents) will also be shown.

\section{THE SQUID MODEL}

A dc SQUID is a superconducting loop interrupted by two junctions. ${ }^{13}$ Since there is a single superconducting loop, the fluxoid quantization, neglecting screening currents, is given by

$$
\phi_{1}-\phi_{2}=2 \pi f,
$$

where $\phi_{1}$ and $\phi_{2}$ are the gauge-invariant phases across the two junctions, and $f=\Phi_{a} / \Phi_{0}$ is the frustration or the ratio between the applied flux $\Phi_{a}$ and the quantum flux $\Phi_{0}=\hbar / 2 e$. Assuming the resistively shunted junction (RSJ) model for the junctions, ${ }^{13}$ the equation of motion of the phase difference is given by the current balance across a branch. In normalized units it reads:

$$
\begin{aligned}
& \ddot{\phi}_{1}+\alpha \dot{\phi}_{1}+\sin \phi_{1}=\gamma, \\
& \ddot{\phi}_{2}+\alpha \dot{\phi}_{2}+\sin \phi_{2}=\gamma .
\end{aligned}
$$

Here time is normalized with respect to the inverse of the Josephson frequency $\omega_{j}=2 \pi I_{0} / C \Phi_{0}(C$ is the capacitance of the junction and $I_{0}$ is the maximum Josephson current), $\alpha=\left(\Phi_{0} / 2 \pi C R^{2} I_{0}\right)^{1 / 2}$ is the damping term ( $R$ is the shunt resistance), and $\gamma$ is the bias current normalized to $I_{0}$. Here and in the following we will assume the parameters of the junctions to be identical.

Taking the sum of Eqs. $(2,3)$ and inserting Eq. (1), the equation of motion for $\phi_{1}$ is readily obtained:

$$
\gamma=\ddot{\phi}_{1}+\alpha \dot{\phi}_{1}+\frac{1}{2}\left[\sin \phi_{1}+\sin \left(\phi_{1}-2 \pi f\right)\right] .
$$

Under the approximation of negligible screening current, the SQUID is described by Eq. (4). Although it is possible to recognize some characteristics of the SQUID, in some respects Eq. (4) is qualitatively incomplete. To better recognize the difference, it is useful to follow the complete derivation of the equation considering also the screening current, ${ }^{15}$ i.e., modifying Eq. (1) as follows:

$$
\phi_{1}-\phi_{2}=2 \pi f+\frac{2 \pi L}{\Phi_{0}} I_{s}
$$

(here $L$ is the inductance of the superconducting loop and $I_{s}$ is the screening current in the loop). What we here call screening current, following Ref. 13, has also been called "mesh current" more recently. ${ }^{9,10}$ Besides the notation, it is important to note that this loop current, while always contributing to the effective magnetic field [i.e., generating a self-field, see Eq. (5)], does not generally screen out completely the magnetic field, as it does in the static case. The current balance now is modified by the presence of the screening current:

$$
\begin{aligned}
& \ddot{\phi}_{1}+\alpha \dot{\phi}_{1}+\sin \phi_{1}=\gamma-\frac{I_{s}}{I_{0}}, \\
& \ddot{\phi}_{2}+\alpha \dot{\phi}_{2}+\sin \phi_{2}=\gamma+\frac{I_{s}}{I_{0}} .
\end{aligned}
$$

Inserting Eq. (5) will lead to two coupled equations rather than one $\left(\beta_{l}\right.$ is the SQUID parameter $\left.\beta_{l}=2 \pi L I_{0} / \Phi_{0}\right)$

$$
\begin{aligned}
& \ddot{\phi}_{1}+\alpha \dot{\phi}_{1}+\sin \phi_{1}=\gamma-\frac{1}{\beta_{l}}\left(\phi_{2}-\phi_{1}\right)+\frac{2 \pi}{\beta_{l}} f, \\
& \ddot{\phi}_{2}+\alpha \dot{\phi}_{2}+\sin \phi_{2}=\gamma+\frac{1}{\beta_{l}}\left(\phi_{2}-\phi_{1}\right)-\frac{2 \pi}{\beta_{l}} f .
\end{aligned}
$$

The most obvious difference between Eqs. (8) and (9) and the $X Y$ model is the increased number of equations: while in the $X Y$ approach the dynamics of the junctions are essentially identical [apart from an additive constant, see Eq. (1)] in the NS context the two dynamics are governed by two coupled differential equations. This remarkable difference is a consequence of the fact that the screening current is a dynamical variable itself, and the fluxoid quantization can be used to eliminate it from the equations in the NS approach, whereas the quantization rule can be used to eliminate a phase variable in the $X Y$ approach. A common characteristic of the two sets of equations is that for $f=0$ a solution is the single-junction free running solution. The difference is that, while for the $X Y$ system this is the only solution, the NS system can allow for other types of solutions, at least for some parameters values. The NS model allows the presence of "propagating solutions," in the sense that an excitation in a junction can propagate to another, giving rise to the socalled "beating solutions." ${ }^{16,17}$ As a consequence, the NS approach will reveal a much richer dynamics than the $X Y$ approach; for instance, it can show hysteresis also in the limit of negligible capacitance (in this limit the second derivative term disappears), while the $X Y$ equation cannot. Another remarkable difference is that for $f=1 / 2$ the $X Y$ equation becomes linear, while the NS set of equations still retains its nonlinear terms.

To get a deeper insight into the difference between the two approaches, we have numerically evaluated $I_{s}$, integrating the Eqs. (8) and (9). To estimate the importance of $I_{s}$ in the dynamics we have plotted the maximum of its absolute value:

$$
I_{s}^{m}=\max \left|I_{s} / I_{0}\right|
$$

Initial conditions are always chosen as $\phi_{1}=\phi_{2}=\sin ^{-1} \gamma$ if $\gamma<1$ and zero otherwise; moreover, $\dot{\phi}_{i}$ was set to zero. We 


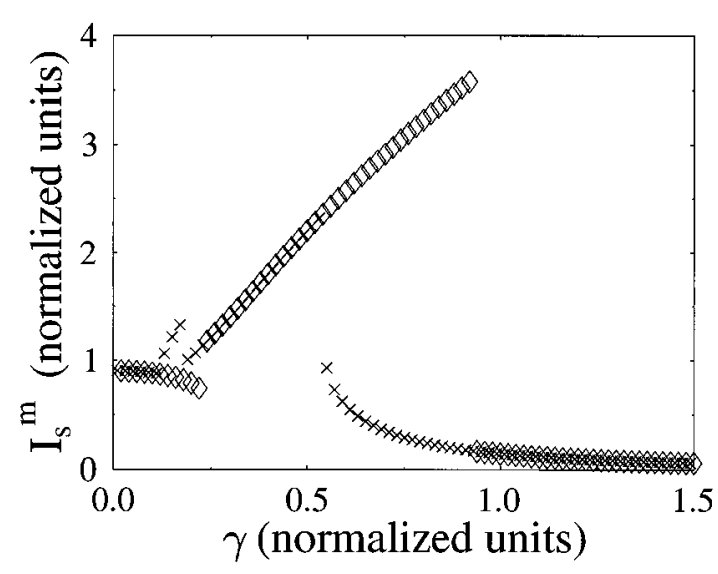

FIG. 1. Maximum screening current vs bias current for a SQUID. Parameters of the simulations are $\alpha=0.25, \beta_{l}=1.0$, and $f=0.5$.

have used two numerical methods (a simple fourth-order Runge Kutta and a more elaborate Bulirsch Stoer) obtaining consistent results. In Fig. 1 we show $I_{s}^{m}$ as a function of the bias current $\gamma$. The two curves are obtained by slowly increasing (diamonds) or decreasing (crosses) the bias current. For both curves there is a region where the screening current can reach significant values. To check that $I_{s}^{m}$ is really a significant test to discriminate where solutions of the NS equations are different from those of the $X Y$ model, we have plotted the microscopic dynamics in Fig. 2 for three points, two for zones of the bias in which $I_{s}^{m}$ is not negligible, and one for higher bias value. It is evident that in the first two cases the difference $\phi_{2}-\phi_{1}$ is not just a constant, as assumed by the $X Y$ model [see Eq. (1)], while this is indeed a good approximation when $I_{s}^{m}$ is negligible.

\section{THE CELL MODEL}

As a first step toward the study of two-dimensional arrays, we will consider in this section the most elementary cell of a square array (see Fig. 3). This cell has already been used to infer the properties of two-dimensional arrays (with a rf bias) by Sohn and Octavio. ${ }^{14}$ A row of such cells has been consid-

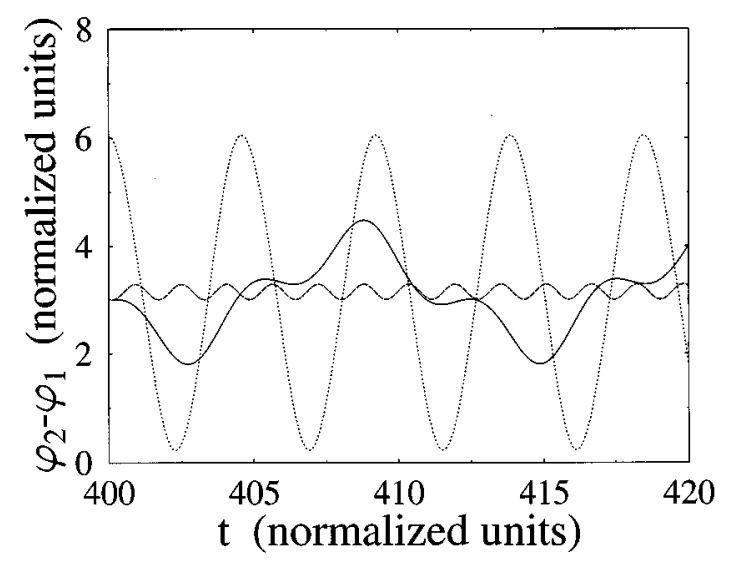

FIG. 2. Dynamics of the phase difference of the two junctions of the SQUID for $\gamma=0.18$ (solid line), $\gamma=0.70$ (short-dotted line), and $\gamma=1.0$ (dotted line). Other parameters are the same as in Fig. 1.

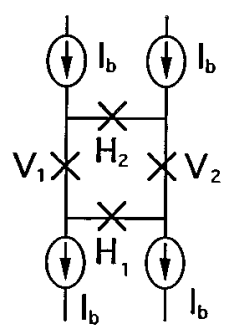

FIG. 3. Schematic of the elementary cell of the two-dimensional square array. Crosses denote the Josephson elements.

ered to investigate the stability of Josephson array. ${ }^{18}$ In the $X Y$ approach the equation for the fluxoid quantization reads (see Fig. 3 for notation):

$$
V_{1}-H_{2}-V_{2}+H_{1}=2 \pi f
$$

Moreover, for the symmetry of the system the following identity also holds: ${ }^{14,18}$

$$
H_{1}=-H_{2}=H
$$

The current balance in the four nodes can be written as $\left(J_{X}\right.$ denotes the current flowing through the RSJ elements of the junction $X$ )

$$
\begin{gathered}
\gamma=J_{H}-J_{V_{1}}, \\
\gamma=-J_{H}-J_{V_{2}}, \\
\gamma=-J_{H}-J_{V_{2}}, \\
\gamma=J_{H}-J_{V_{1}} .
\end{gathered}
$$

Taking the sum and difference of the independent equations, the dynamics of the cell in the $X Y$ model is governed by the following two equations: ${ }^{14}$

$$
\begin{gathered}
\ddot{S}+\alpha \dot{S}+2 \sin \left(\frac{1}{2} S\right) \cos \left(\frac{1}{2} D\right)=-2 \gamma, \\
\ddot{D}+\alpha \dot{D}+\sin \left(\frac{1}{2} D\right) \cos \left(\frac{1}{2} S\right)+\sin \left(\frac{1}{2} D-\pi f\right)=0,
\end{gathered}
$$

where $S=V_{1}+V_{2}$ and $D=V_{1}-V_{2}$.

For the NS approach, as usual, we have to consider also the screening current, while for the same symmetry reasons Eq. (12) still holds. In conclusion Eq. (11) becomes

$$
V_{1}-V_{2}+2 H=2 \pi f-\frac{2 \pi L I_{s}}{\Phi_{0}}
$$

and the equations of motion of the cell are 


$$
\begin{gathered}
\ddot{V}_{1}+\alpha \dot{V}_{1}+\sin V_{1}=-\gamma+\frac{1}{\beta_{l}}\left(V_{1}-V_{2}+2 H\right)-\frac{2 \pi}{\beta_{l}} f, \\
\ddot{V}_{2}+\alpha \dot{V}_{2}+\sin V_{2}=-\gamma-\frac{1}{\beta_{l}}\left(V_{1}-V_{2}+2 H\right)+\frac{2 \pi}{\beta_{l}} f, \\
\ddot{H}+\alpha \dot{H}+\sin H=\frac{1}{\beta_{l}}\left(V_{1}-V_{2}-2 H\right)-\frac{2 \pi}{\beta_{l}} f .
\end{gathered}
$$

For comparison we write also Eqs. (20)-(22) in terms of the variables $S$ and $D$; in this case the equations yield

$$
\begin{gathered}
\ddot{S}+\alpha \dot{S}+2 \sin \left(\frac{1}{2} S\right) \cos \left(\frac{1}{2} D\right)=-2 \gamma, \\
\ddot{D}+\alpha \dot{D}-2 \cos \left(\frac{1}{2} S\right) \sin \left(\frac{1}{2} D\right)=\frac{2}{\beta_{l}}(D+2 H)-\frac{4 \pi}{\beta_{l}} f, \\
\ddot{H}+\alpha \dot{H}+\sin H=\frac{1}{\beta_{l}}(D+2 H)-\frac{2 \pi}{\beta_{l}} f .
\end{gathered}
$$

Equations (23) and (17) are identical, but Eq. (18) is not easily recognized as an approximations of Eqs. (24) and (25). Therefore, the considerations done for the SQUID also apply here: the two approaches differ in the number of equations, and it seems difficult to predict a priori for which parameter values the screening current is negligible. Rather, we numerically integrate Eqs. (20)-(22) and in Fig. 4 we show the dependence of $I_{s}^{m}$ on the parameter $\beta_{l}$ as a function of the bias current for two different values of the damping coefficient $\alpha$; in Fig. 5 is shown the behavior of the screening current as a function of the frustration and the bias current, for the same values of the damping coefficient. The results shown in these figures can be summarized as follows:

(1) For $\gamma>>1$ the screening current decreases. This corresponds to the observation that (in the overdamped limit) for high bias current the solutions are nearly sinusoidal (plus a constant slope) and with a constant phase shift in presence of magnetic field. ${ }^{18}$ This state corresponds to negligible screening current.

(2) For high inductance values (for instance when $\beta_{l} \geqslant 2$ for $\alpha=0.25$ and $f=0.5$ ) the screening current is also negligible. This is not surprising because for high values of the inductance the current induced by the external field is small. On the contrary, for the same values of $\alpha$ and $f$ and for $\beta_{l}$ values down to 0.1 (a fairly low value for practical arrays ${ }^{19}$ ), the screening current increases, up to values where it is not negligible; however, for $\beta_{l}<0.1$ this region shrinks. As for the value of $\beta_{l}$ actually used in the experiments it varies from $\beta_{l}=0.062$ (Ref. 20) to $\beta_{l}=0.8$ (Ref. 21).

(3) The capacitance and the dissipation play an important role in controlling the screening current effect. Significant screening current are observed for $\alpha<1$, i.e., for relatively high values of the capacitance or for low values of the dissipation.

It is also important to notice that, as expected, hysteresis plays an important role. In Figs. 4 and 5 we have always
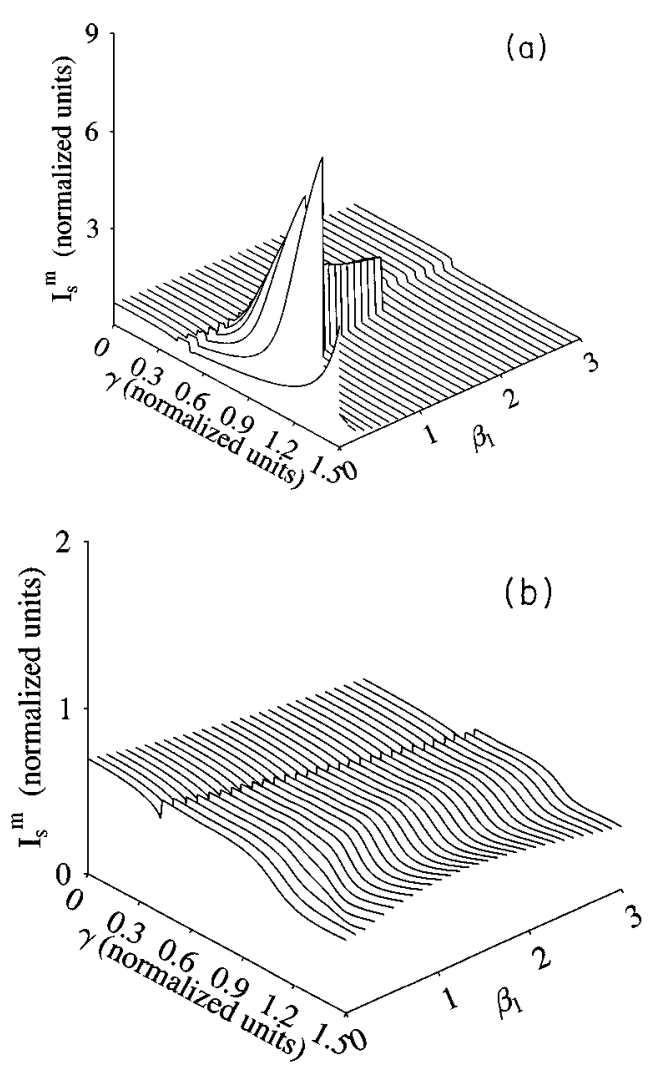

FIG. 4. Three-dimensional (3D) plot of the maximum of the screening current $I_{s}^{m}$ vs the bias current $\gamma$ and the SQUID parameter $\beta_{l}$ for the elementary cell for (a) $\alpha=0.25$ and (b) $\alpha=1.0$. The frustration is set to $f=0.5$.

used as initial conditions all the phases and phase derivatives equal to zero, therefore no hysteresis is shown. On the other hand, as mentioned for Fig. 1, hysteresis does exist in the system. Also in the cell simulations of the underdamped case for a different choice of the initial conditions there are solutions, not shown in Fig. 5(a), that exhibit a nonzero screening current. It is also noteworthy that in Fig. 5(a) the behavior of the screening current is irregular. The irregularity is caused, in fact, by hysteresis.

As we have pointed out in the introduction, twodimensional arrays are of interest if a large number of junctions can be locked together. Even if we believe that the simple cell studied in the preceding section offers the distinct advantage of simplicity and illustrates the basic mechanism, it is quite natural to ask if it can also furnish quantitative predictions on larger arrays. To check that the results obtained so far are not crucially dependent on the fact that we are considering only an elementary cell, we have investigated the behavior of larger arrays in few cases, using the NS equations. ${ }^{8}$ In Fig. 6 the behavior of a $10 \times 10$ array is shown where zones of screening current comparable with the elementary cell can be clearly seen; in these zones the overall dynamics can be directly compared with that of the elementary cell (see also the discussion below). These zones corresponds to a large range of the parameters; however, besides these zones in the low damping case $[\alpha=0.25$, see Fig. $6(\mathrm{a})$ ], a region of very large maximum screening current is shown: this region corresponds to the penetration of static fluxons in the array and does not have any correspondence in 

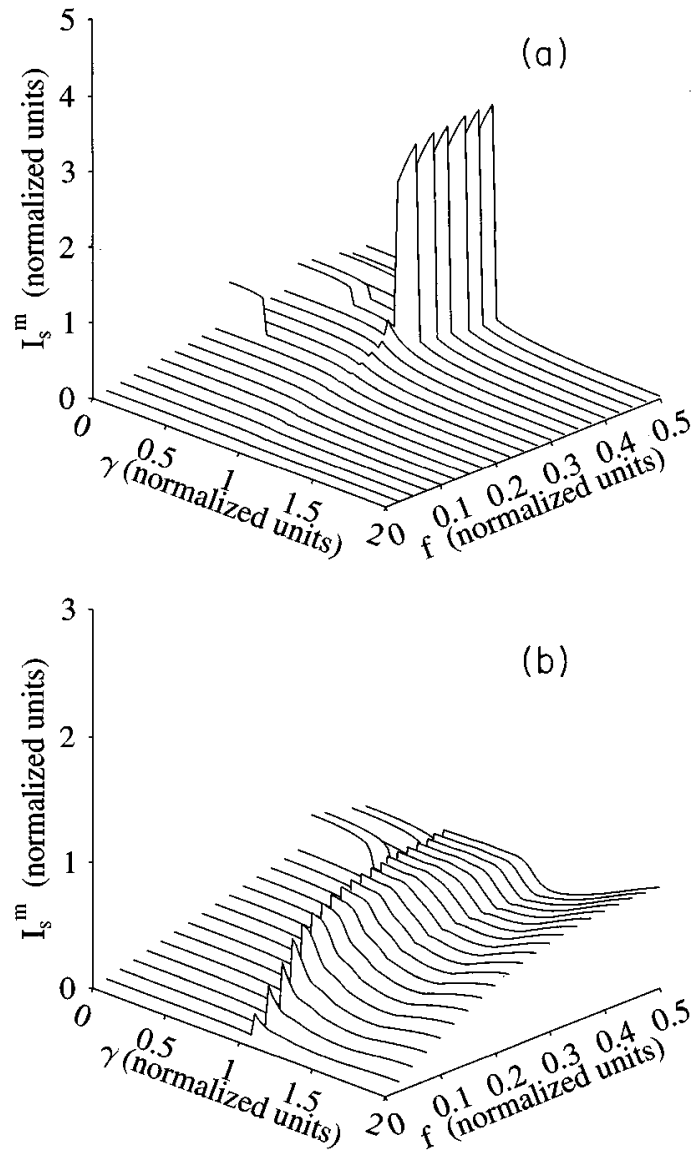

FIG. 5. 3D plot of the maximum of the screening current $I_{s}^{m}$ vs the bias current $\gamma$ and the frustration $f$ for the elementary cell for (a) $\alpha=0.25$ and (b) $\alpha=1.0$. The SQUID parameter is set to $\beta_{l}=1.0$.

the cell case; further studies of this region will be carried out in the future.

\section{MICROSCOPIC DYNAMICS}

So far we have dealt only with the "macroscopic" quantity $I_{s}^{m}$. Although we claim that this is sufficient to discriminate between states where model (1) and model (2) do not give consistent results (because the set of equations are the same if $I_{s}$ is negligible) it is interesting to investigate in which sense the dynamics differ when the screening current is not negligible. For sake of simplicity we will concentrate on the microscopic dynamics in the elementary square cell.

We have systematically found that when the screening current is not negligible there appear solutions that are close to the well known beating solutions for SQUID's, ${ }^{16,17}$ i.e., to solutions that correspond to the entry and propagation of fluxons across the cell or to the motion of fluxons across the array. To recognize this, we have shown in Fig. 7 the maximum screening current as a function of the voltage behavior together with the IV characteristic. It is evident that a large screening current is associated to a resonant step that is reminiscent of the Fiske steps in long Josephson-junctions. In fact, in the long Josephson-junction language resonant structures are called Fiske steps if they occur in the presence of a magnetic field and zero-field steps if they occur in absence of magnetic field. ${ }^{13}$
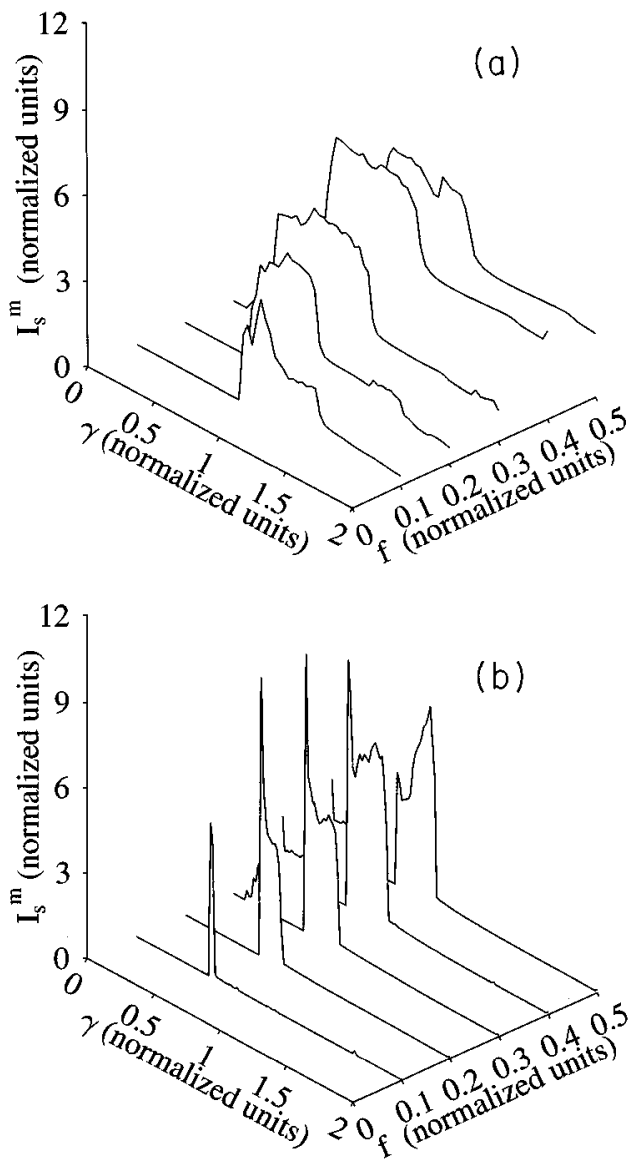

FIG. 6. Maximum screening current as a function of $\gamma$ and $f$ for an array $10 \times 10$ with $\beta_{l}=1$. Parameters of the simulations are (a) $\alpha=0.25$, and (b) $\alpha=1.0$.

It is also evident that there is a sharp change in the behavior of $I_{s}^{m}$ when the system switches from the resonant step to another solution. To better understand the nature of this transition, we have plotted in Fig. 8 the voltage across the two vertical junctions for two values of the bias current: just before the switch and just after. The difference between the two dynamics is that, while on the resonant step there is a large voltage pulse, after the switch the dynamics is much

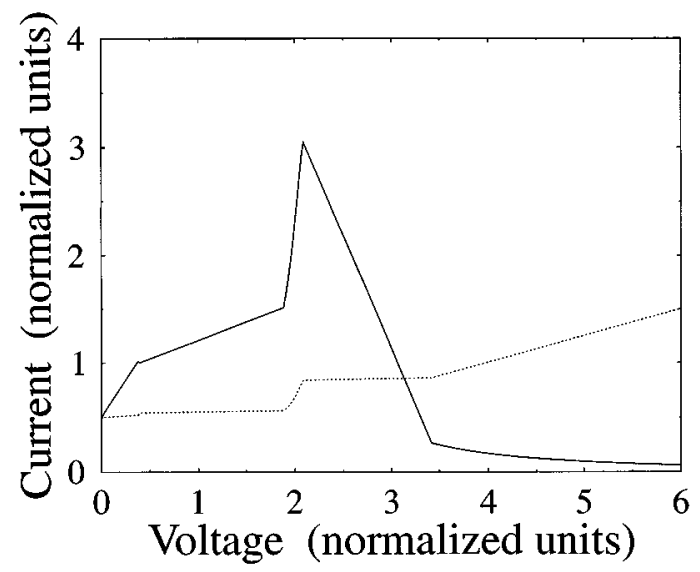

FIG. 7. Voltage (dotted line) and of $I_{s}^{m}$ (solid line) as a function of the bias current in the square cell. Parameters of the simulations are: $\alpha=0.25, \beta_{l}=1$, and $f=0.5$. 


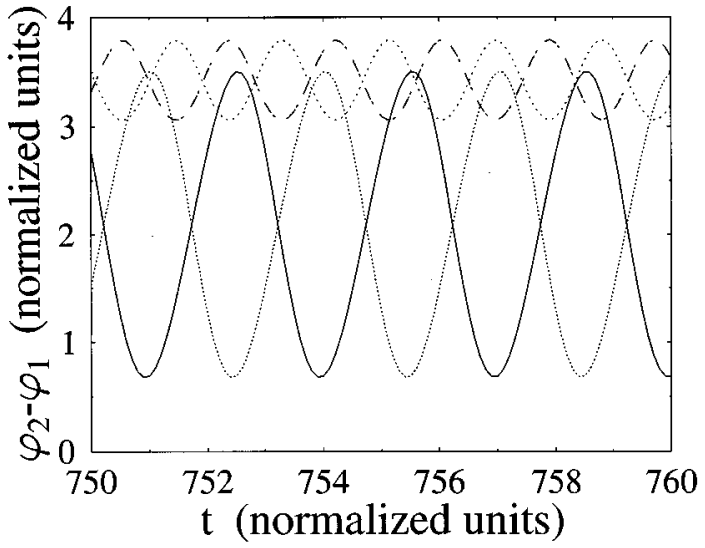

FIG. 8. Dynamics of the voltage across the vertical junctions in the square cell for $\gamma=0.84$ (solid line and dotted line) and $\gamma=0.86$ (dotted lines with shorter length). Parameters of the simulations are the same as Fig. 7.

more uniform with a smaller modulation of the voltage. We claim that the transition is similar to that observed for onedimensional arrays or for long Josephson junctions. ${ }^{22,23}$

A comparison between Figs. 1 and 2 and Figs. 7 and 8 strongly indicates that the dynamics are very similar to that observed for the simple SQUID: in fact, as in the SQUID case, resonant states appear, in general, together with high values of screening currents, and, similarly, the dynamics appears to be uniform where screening currents are negligible.

The different nature of the two solutions can be clearly seen in Fig. 9, where we show a plot of the time delay across the two vertical junctions of two peaks of voltage. Indeed, while on the resonant step this time delay roughly tends to a constant (corresponding to the maximum speed of propagation of a signal across the system), in the state where the screening currents are negligible the speed increases with current. This suggests that in the latter mode there is no actual signal propagating across the cell but rather a modulated solution similar to that obtained in Ref. 18 for overdamped junctions in the high bias limit. It should be noticed

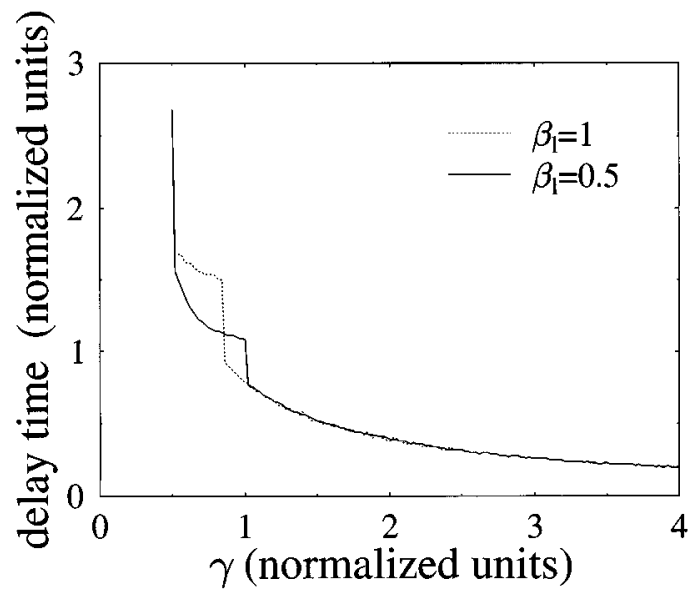

FIG. 9. Time delay between two peaks of the voltage in two adjacent vertical junctions for $\beta_{l}=1$ (plusses) and $\beta_{l}=0.5$ (stars). Parameters of the simulations are $\alpha=0.25$ and $f=0.5$. that the very concept of a localized solution propagating across the array is not applicable to the solution arising from the $X Y$ model. If we adopt as a working definition of propagating fluxon an excitation of roughly one flux quantum moving from a cell to another, then to neglect screening currents prevents the appearance of such solutions because the magnetic field is supposed to be uniform across the array. For these solutions, while it is still possible to identify their center, the field is spread across the whole array: in this sense it is not unphysical that the propagation speed, being a phase velocity, can be infinity (see Fig. 9). Since the $X Y$ model does not allow signal propagation, we conclude that it can be an approximation for the NS equations (as well as any model including screening current effects through mutual inductances) only if the latter do not carry a localized solution propagating across the array.

Since the uniform regime occurs for higher bias, an interesting question is if the border between those two regions corresponds to the border between the regions of flux motion and uniform solutions devised by Lachenmann et al. with the low-temperature scanning electron microscopy (LTSEM) technique. ${ }^{24}$ When comparing the thresholds obtained numerically with those observed experimentally it is quite arbitrary to decide how small the screening current should be to lead to the disappearance of the signal measured by the LTSEM; therefore a detailed quantitative comparison is not possible on the basis of the results shown here. However, the measured threshold for an array $10 \times 10$ critically damped $(\alpha \simeq 1)$ is $\gamma \simeq 4,{ }^{24}$ a region where our simulations predict that the screening current is small: $I_{s}^{m} \simeq 0.16$ or roughly $4 \%$ of the bias current at most, i.e., for $f=0.5$.

It is nevertheless possible to notice that both the screening current and the LTSEM signal indicates an homogeneous solution for high bias current. ${ }^{24}$ If the hypothesis that the two thresholds are related is true, it is possible to go further and to speculate that the coherent emission from Josephsonjunctions arrays is related to the presence of non-negligible screening currents.

\section{CONCLUSIONS}

We have proven that the choice of the more appropriate model for Josephson-junction arrays depends on the parameters, especially on the bias current and the SQUID parameter $\beta_{l}$. It is possible, in fact, to show numerically that for certain regions of the parameters the contribution to flux quantization arising from the screening current, neglected in the so-called $X Y$ model, is important, and this can happen also for relatively small values of the inductance of the loop. We have traced the origin of this to the structure of the equations, noticing that the screening current is a dynamical variable itself whose value cannot be easily predicted a priori, and we were able to investigate its behavior only numerically. We have also found that the presence of a nonnegligible screening current prevents the occurrence of uniform solutions to set in and rather induces solutions that are known, in the context of SQUID's, as beating solutions. ${ }^{16,17}$ Finally, there are reasons to believe that there might be a connection between the presence of significant screening currents and the phenomenon of "row switching" observed with the technique of LTSEM. ${ }^{24}$ It is worth recalling that we have (a) neglected any spread of the parameters and (b) retained self-inductances only and neglected mutual inductances. 


\section{ACKNOWLEDGMENTS}

We thank P. Carelli and N.F. Pedersen for a critical reading of this work and T. Doderer, S.G. Lachenmann, A. Laub, P.L. Christiansen, and G. Kalosakas, for valuable discus- sions. The financial support of ESPRIT Project No. 7100, SCIENCE Program No. SCI*-CT91-0760 "Coupled Josephson junctions," the Human Capital and Mobility grant, Program No. ERBCHRXCT 920068, and of the Progetto Finalizzato "Superconductive and Cryogenics Technologies" are gratefully acknowledged.
${ }^{1}$ D.R. Tilley, Phys. Lett. 33, 205 (1970).

${ }^{2}$ K. Wiesenfeld, S.P. Benz, and P.A.A. Booi, J. Appl. Phys. 76, 3835 (1994).

${ }^{3}$ R.D. Parmentier, Acta Phys. Slovaca 44, 303 (1994).

${ }^{4}$ D. Dominguez and V. Josè, Int. J. Mod. Phys. B 8, 3749 (1994).

${ }^{5}$ S. Teitel and C. Jayaprakash, Phys. Rev. B 27, 2598 (1983).

${ }^{6}$ T.C. Halsey, Phys. Rev. B 31, 5728 (1985).

${ }^{7}$ N. Groenbech-Jensen, A.R. Bishop, F. Falo, and P.S. Lomdahl, Phys. Rev. B 45, 10139 (1992).

${ }^{8}$ K. Nakajima and Y. Sawada, J. Appl. Phys. 52, 5732 (1981).

${ }^{9}$ J.R. Phillips, H.S.J. Van der Zant, J. White, and T.P. Orlando, Phys. Rev. B 47, 5219 (1993).

${ }^{10}$ D. Reinel, W. Dietrich, T. Wolf, and A. Majhofer, Phys. Rev. B 49, 9118 (1994).

${ }^{11}$ A Majhofer, T. Wolf, and W. Dieterich, Phys. Rev. B 44, 9634 (1991).

${ }^{12}$ S.P. Benz, M.S. Rzchowski, M. Tinkham, and C.J. Lobb, Phys. Rev. Lett 64, 692 (1990).

${ }^{13}$ A. Barone and G. Paternò, Physics and Application of the Josephson Effect (Wiley, New York, 1982).

${ }^{14}$ L.L. Sohn and M. Octavio, Phys. Rev. B 49, 9236 (1994).

${ }^{15}$ C.D. Tesche and J. Clarke, J. Low Temp. Phys. 29, 301 (1977).

${ }^{16}$ E. Ben-Jacob and Y. Imry, J. Appl. Phys. 526806 (1981).
${ }^{17}$ J.A. Ketoja, J. Kurkijärvi, and R.K. Ritala, Phys. Rev. B 30, 3757 (1984).

${ }^{18}$ G. Filatrella, and K. Wiesenfeld, J. Appl. Phys. 78, 1878 (1995).

${ }^{19}$ This limit, as mentioned in the Introduction, refers to possibility of using practical arrays as coherent sources of EM radiation rather than the limit set on the fabrication that is at about $\beta_{l} \sim 0.001$ (essentially because of the stray inductance of the junctions in the array).

${ }^{20}$ H.S.J. Van Der Zant, Ph.D. thesis, Technische Universiteit, Delft, 1991.

${ }^{21}$ S.P. Benz and C.J. Burroughs, Appl. Phys. Lett. 58, 2162 (1991).

${ }^{22}$ R.D. Parmentier, in The New Superconducting Electronic, edited by H. Weinstock and R.W. Ralston (Kluwer, Dordrecht, 1993), p. 221.

${ }^{23}$ A.V. Ustinov, M. Cirillo, B.H. Larsen, A. Oboznov, P. Carelli, and G. Rotoli, Phys. Rev. B 51, 3081 (1995).

${ }^{24}$ S.G. Lachenmann, T. Doderer, D. Hoffmann, P.A.A. Booi, and S.P. Benz, Phys. Rev. B 50, 3158 (1994); T. Doderer, S.G. Lachenmann, and R.P. Huebener, in Proceedings of Macroscopic Quantum Phenomena and Coherence in Superconducting Arrays, Frascati, 1995, edited by C. Giovannella and M. Tinkham (World Scientific, Singapore, 1996). 